\title{
Covid-19-associated acute haemorrhagic leukoencephalomyelitis
}

\author{
Rahul Handa ${ }^{1}$ (I) Satyan Nanda ${ }^{1} \cdot$ Atul Prasad $^{1} \cdot$ Rajiv Anand $^{1} \cdot$ Dhruv Zutshi $^{1} \cdot$ Sujata K. Dass ${ }^{1}$. \\ Prabhjeet Kaur Bedi ${ }^{1}$ - Aarti Pahuja ${ }^{1}$ - Pankaj Kumar Shah ${ }^{1}$ • Bipan Sharma ${ }^{1}$
}

Received: 30 July 2020 / Accepted: 29 August 2020 / Published online: 2 September 2020

(C) Fondazione Società Italiana di Neurologia 2020

\section{Dear Editor,}

Neurological symptoms in Covid-19 involving central and peripheral nervous system have been reported with varying frequency. The importance of identifying neurological symptoms cannot be overemphasised by the fact that loss of sense of smell is now considered one of the earliest features of Covid-19 [1]. Although impaired consciousness has been reported in approximately $17 \%$ patients with Covid-19, acute haemorrhagic leukoencephalomyelitis (AHL) as its cause has been reported in very few cases [2]. AHL is characterised by rapidly progressive, fulminant inflammatory haemorrhagic demyelination mainly involving the white matter and is almost always monophasic. Aetiology is most likely post infectious and is usually associated with poor prognosis [3]. We herein report a case of acute haemorrhagic leukoencephalomyelitis secondary to Covid-19 from a tertiary care centre in India. To the best of our knowledge, this is the first case of Covid-19-associated AHL to be reported from India.

\section{Case report}

A 33-year-old male, known case of chronic kidney disease since 5 years (on maintenance haemodialysis: 3/week since 1 year) and hypertension was admitted to our hospital with chief complaints of fever for 5 days, acute onset rapidly progressive weakness of both upper and lower limbs since 3 days and altered sensorium since 1 day. Patient had an episode of generalised tonic-clonic seizures in the emergency and was started on injection lacosamide. On examination, patient was haemodynamically stable, tachypnoeic with respiratory rate of

Rahul Handa

rahulhanda0411@gmail.com

1 Department of Neurology, BLK Super Speciality Hospital, New Delhi, India 30/minute and Glasgow coma scale (GCS) score as E2 V1 M4. Brainstem reflexes were well elicitable. Deep tendon reflexes were absent in all four limbs with bilateral extensor plantar response. No neck rigidity was appreciable. He was intubated in view of poor GCS to protect the airway and to provide respiratory support. A clinical diagnosis of encephalitis was kept, and patient was started on acyclovir, ceftriaxone, lacosamide and other supportive treatment. Routine blood investigations showed normocytic normochromic anaemia ( $\mathrm{Hb}: 8.8 \mathrm{~g} \%)$ with elevated urea $(84.20 \mathrm{mg} / \mathrm{dL})$ and creatinine $(6.74 \mathrm{mg} / \mathrm{dL})$. Chest X-ray findings were consistent with bilateral hilar dominant opacities. Inflammatory markers were elevated, i.e. interleukin (IL-6), $8.30 \mathrm{pg} / \mathrm{ml}$ (normal 0-7); D dimer, $0.98 \mathrm{mcg} / \mathrm{ml}$ (normal: 0-0.5); and S. ferritin levels, 2973 ng/ml (normal: 21.8-274). Covid-19 PCR test from nasopharyngeal swab was positive. USG abdomen showed changes consistent with medical renal disease with no other noteworthy finding. Patient's electroencephalogram (EEG) was suggestive of diffuse background slowing with no epileptiform discharges.

In view of altered sensorium, a brain magnetic resonance imaging (MRI) was advised which revealed symmetrical FLAIR hyperintensities involving bilateral subcortical fronto-parietal lobes, splenium of corpus callosum, medulla and visualised cervical cord with petechial haemorrhages and evidence of diffusion restriction involving splenium of corpus callosum (Figs. 1, 2, 3 and 4). Following the MRI, a lumbar puncture was done to rule out any infective cause explaining the above MRI changes. Cerebrospinal (CSF) fluid examination revealed normal protein, $35 \mathrm{mg} \%$; sugar, 75 $\mathrm{mg} \%$; and cell count, 5 cells (all lymphocytes). Viral RTPCR panel (including herpes simplex 1 and 2) and Gene Xpert for tuberculosis and cryptococcal antigen were negative. Thus, a final diagnosis of acute haemorrhagic leukoencephalomyelitis secondary to Covid-19 was made, and patient was started on methyl prednisolone $1 \mathrm{gm} \mathrm{IV} \mathrm{per}$ day for 5 days. This resulted in good neurological improvement of the patient as he became conscious and responsive to verbal commands. He started showing improvement in motor 


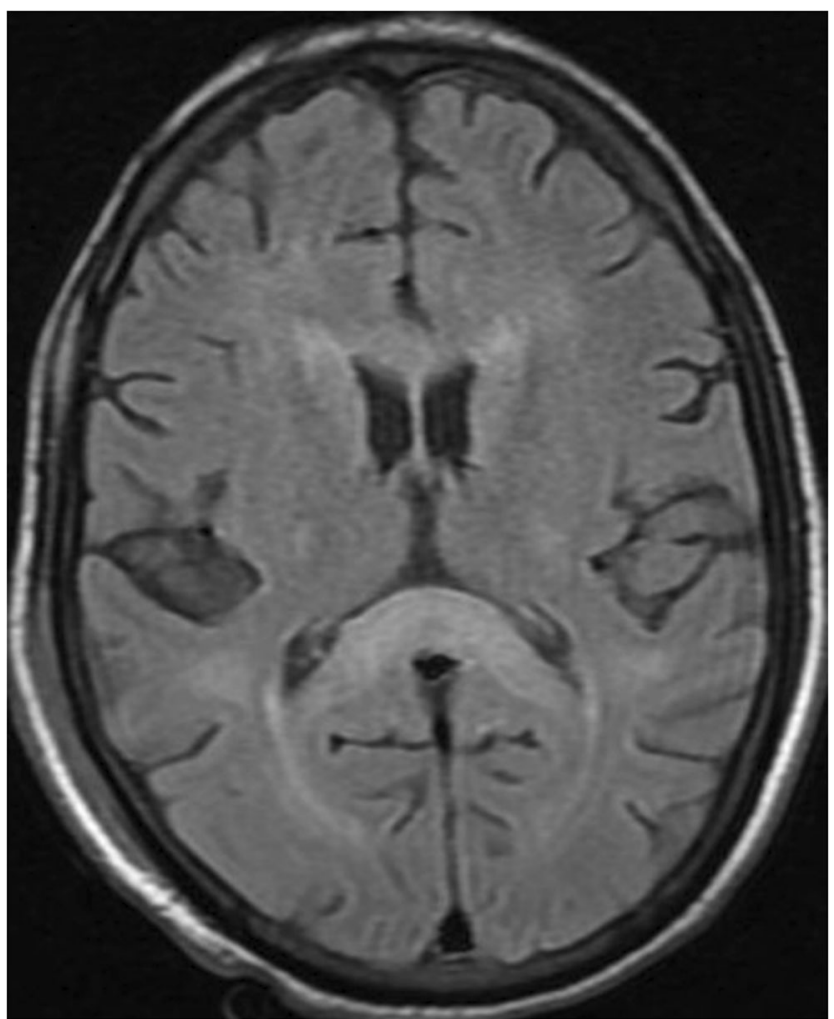

Fig. 1 MRI FLAIR axial image showing hyperintensity in splenium of corpus callosum

deficits too with power in upper and lower limbs improving to grade $3 / 5$ as per Medical Research Council (MRC) grading by 5 th day of IV steroid therapy. But his respiratory features and $\mathrm{X}$-ray findings continued to deteriorate, and his ventilatory requirement progressively increased. At day 10 of hospitalisation, he was not able to maintain adequate oxygen saturation in spite of full ventilatory support and subsequently went into shock followed by cardiac arrest.

\section{Discussion}

Possible mechanism by which Covid-19 affects nervous system is still under study, but few hypotheses include direct damage to receptors, cytokine-related injury, retrograde travel along the nerve fibres and immune mediated. Mechanism of encephalitis in patients with Covid-19 is not totally clear, but likely possibilities include inflammatory injury and direct viral infection [4]. A number of case series have shown association of CNS manifestations with severe Covid-19 infection and elevated inflammatory markers like D dimer and interleukin6 (IL-6) $[5,6]$. Our case report supports the hypotheses of association between CNS manifestation and severe form of Covid-19 infection with raised inflammatory markers.

AHL is one of the aggressive variants of acute disseminated encephalomyelitis (ADEM), which is found in less

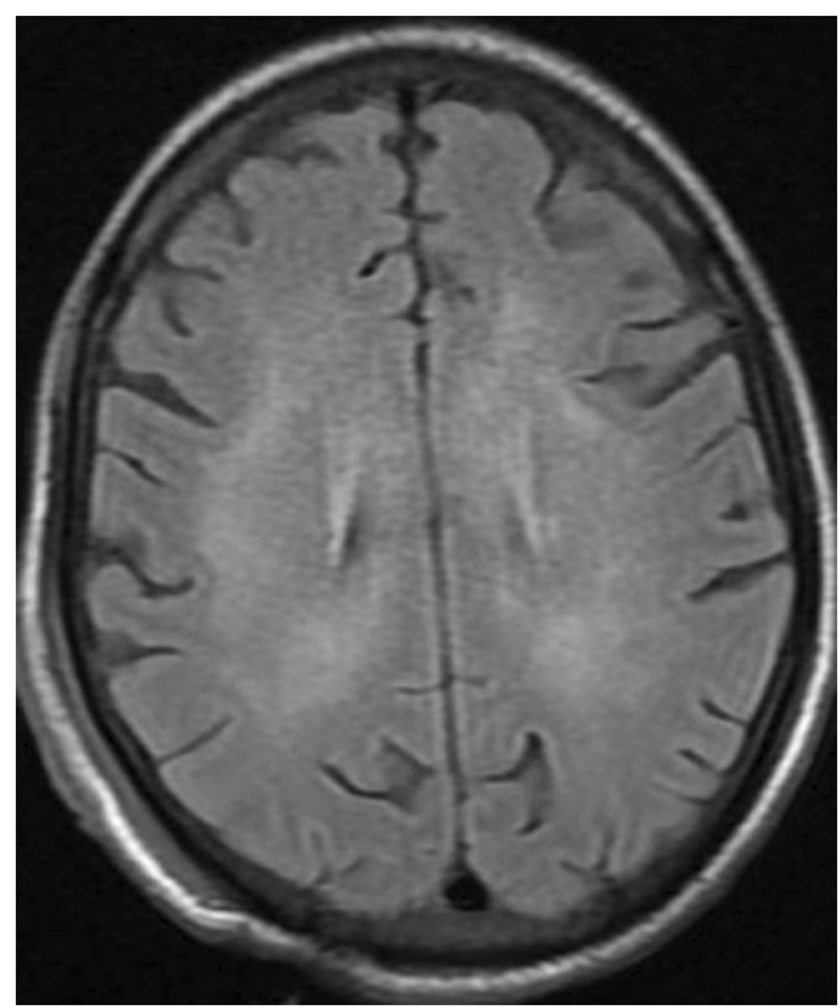

Fig. 2 MRI FLAIR axial image showing confluent hyperintensities involving bilateral subcortical posterior fronto-parietal lobes

than $2 \%$ of patients with ADEM and is most often triggered by an upper respiratory tract infection [4]. A few clinical and radiological clues help in differentiating ADEM from its rare and more aggressive variant. Although AHL and ADEM share a common aetiology, i.e. autoimmune process directed against CNS myelin, clinically, hyperacute onset, rapid progression and high mortality seen in AHL help in differentiating it from ADEM [7]. Similarly, both ADEM and AHL on imaging are characterised by perivenular demyelination and inflammation predominantly involving white matter, but asymmetric or symmetric subcortical white matter involvement of posterior fronto-parietal lobes with relative sparing of the cortex is characteristic of AHL. Involvement of basal ganglia which is quite common in ADEM is relatively rare in AHL and thus helps in differentiating the two [8]. Our patient had an almost sudden onset and a very rapidly progressive downhill course associated with MRI showing bilateral symmetric confluent FLAIR hyperintensities in subcortical white matter involving the posterior fronto-parietal lobes and splenium of corpus callosum suggestive of demyelination and inflammation, with sparing of basal ganglia and cortex with punctate haemorrhages noted in the splenium. FLAIR hyperintensity in cervical cord was suggestive of myelitis. Thus, clinico-radiological manifestation of our patient was suggestive of AHL, and although it has a poor prognosis, 


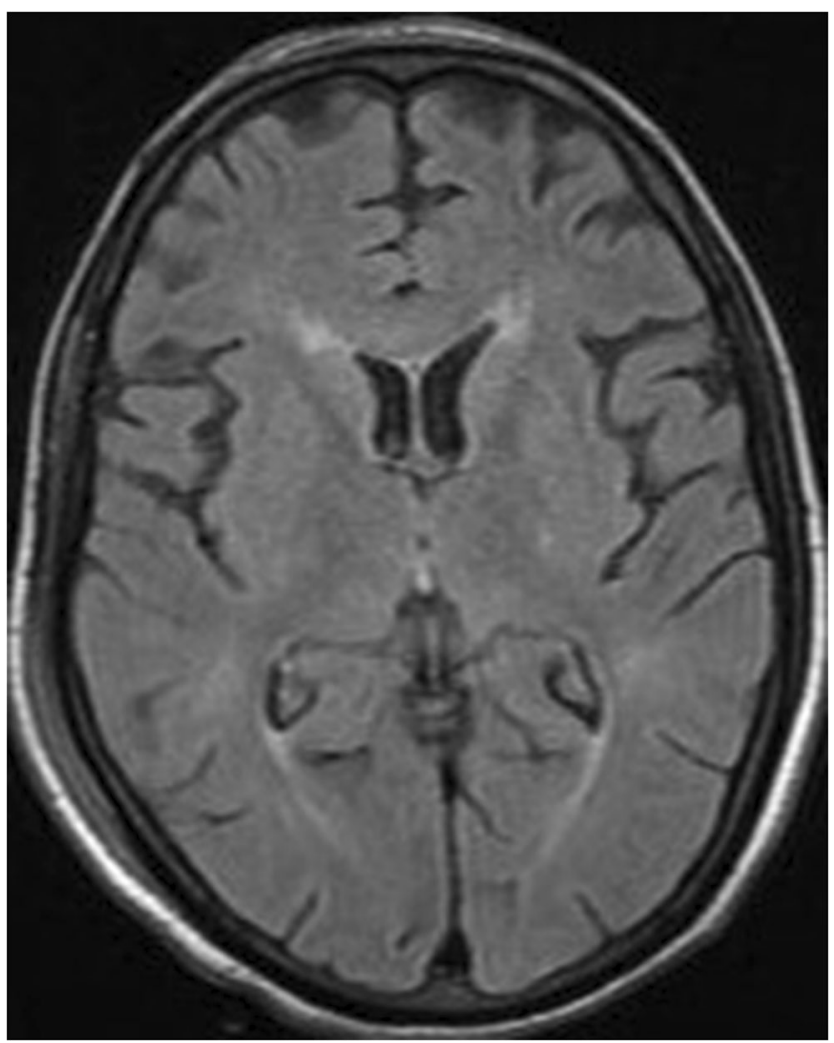

Fig. 3 MRI FLAIR axial image showing sparing of basal ganglia

our patient had a good neurological response to steroids, but unfortunately, severe respiratory dysfunction due to Covid-19 led to his mortality. Patients with chronic kidney disease who develop Covid-19 infection have increased risk of severity and mortality as compared with

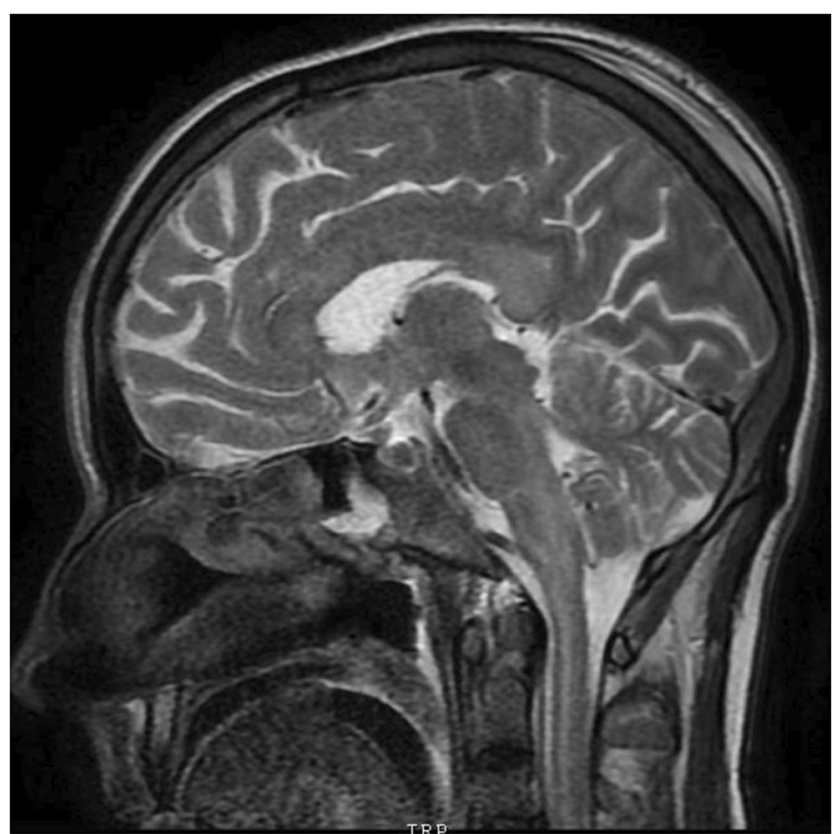

Fig. 4 MRI T2W sagittal image showing hyperintensity involving medulla and cervical cord general population [9]. Thus, chronic kidney disease may have played an important role in clinical deterioration of the patient. Other conditions which share the common imaging findings are progressive multifocal leukoencephalopathy (PML), vasculitis and lymphoma. Hyperacute onset and rapidly progressive course help in differentiating the above conditions from AHL, as all of these have a subacute to indolent course [8]. Various treatments which have been described to be effective in AHL include high dose steroids, IV Immunoglobulins, plasmapheresis and cyclophosphamide [3].

\section{Conclusion}

As there is huge amount of research going on in recognising new clinical presentations of Covid-19, evidence favouring neurological manifestations in Covid-19 is increasing with each passing day. AHL is a fulminant disease characterised by hyperacute onset and rapidly deteriorating course with almost certain mortality if not treated early and aggressively. Thus, it is imperative for the emergency and respiratory physicians to be aware of this condition as mostly they are the ones who initially come in contact with the Covid-19 patients Early diagnosis and aggressive treatment would greatly help in improving the prognosis and reducing the morbidity in this otherwise fatal disease.

\section{Compliance with ethical standards}

Conflict of interest The authors declare that they have no conflict of interest.

\section{References}

1. Özdağ Acarli AN, Samanci B, Ekizoğlu E, Çakar A, Şirin NG, Gündüz T, Parman Y, Baykan B (2020) Coronavirus disease 2019 (COVID-19) from the point of view of neurologists: observation of neurological findings and symptoms during the combat against a pandemic. Noro Psikiyatr Ars 57(2):154-159

2. Markus R, Brew BJ, Turner J, Pell M (1997) Successful outcome with aggressive treatment of acute haemorrhagic leukoencephalitis. J Neurol Neurosurg Psychiatry 63(4):551-552

3. Marin SE, Callen DJ (2013) The magnetic resonance imaging appearance of monophasic acute disseminated encephalomyelitis: an update post application of the 2007 consensus criteria. Neuroimaging Clin N Am 23(2):245-266

4. Bridwell R, Long B, Gottlieb M (2020) Neurologic complications of COVID-19. Am J Emerg Med 38(7):1549.e3-1549.e7

5. Mao L, Jin H, Wang M et al (2020) Neurologic manifestations of hospitalized patients with coronavirus disease 2019 in Wuhan, China [published online ahead of print, 2020 Apr 10]. JAMA Neurol 77(6): $1-9$

6. Li Z, Liu T, Yang N et al (2020) Neurological manifestations of patients with COVID-19: potential routes of SARS-CoV-2 
neuroinvasion from the periphery to the brain [published online ahead of print, 2020]. Front Med:1-9

7. Vartanian TK, Monte S (1999) Weekly clinicopathological exercises. Case1-1999. N Engl J Med 340:127-135

8. Kuperan S, Ostrow P, Landi MK, Bakshi R (2003) Acute hemorrhagic leukoencephalitis vs ADEM: FLAIR MRI and neuropathology findings. Neurology. 60(4):721-722
9. Oyelade T, Alqahtani J, Canciani G (2020) Prognosis of COVID-19 in patients with liver and kidney diseases: an early systematic review and meta-analysis. Trop Med Infect Dis 5(2):80

Publisher's note Springer Nature remains neutral with regard to jurisdictional claims in published maps and institutional affiliations. 